\title{
Harnessing D2D Communications in Collaborative Mobile Clouds for Content Sharing: An Energy Efficient Communication Scheme
}

\author{
Jun Huang ${ }^{1}$, Chao Huang ${ }^{2}$, Cong-cong Xing ${ }^{3}$ \\ \{xiaoniuadmin@gmail.com, cong-cong.xing@nicholls.edu $\}$ \\ ${ }^{1}$ School of CS, Chongqing Univ. of Posts and Telecom, Chongqing, China 400065 \\ ${ }^{2}$ School of Commu. and Info. Eng., Chongqing Univ. of Posts and Telecom, Chongqing, China \\ 400065 \\ ${ }^{3}$ Nicolls State Unviersity, 906 East 1st St. Thibodaux, Louisiana, USA 70301
}

\begin{abstract}
This paper presents an energy efficiency (EE) communication scheme for content sharing with Collaborative Mobile Clouds (CMC) and D2D cluster. A waterfilling-based data segmentation approach for content distribution is proposed. Within the $\mathrm{CMC}$, the cost-effective resource allocation and the power control mechanisms for D2D communications are designed. Through performance comparisons, it is disclosed that the proposed scheme dominates different variations of it in terms of system EE.
\end{abstract}

Keywords: Energy efficiency, Collaborative Mobile Cloud, D2D communications, Content Sharing.

\section{Introduction}

Although the paradigm of Device-to-Device (D2D) communications has been introduced as a complementary technology to the $5 \mathrm{G}$ mobile system, it also brings in challenges when the radio resources are being reused by both cellular and D2D links. Among all these challenges, while interference coordination has long been the major one in D2D research, energy efficiency (EE) of mobile systems plays a crucial role, especially in 5G cellular networks featured with higher data rate, lower latency, and much richer contents [1]. This situation results in the necessity of the reconsideration of D2D communication patterns and the corresponding resource management schemes.

Grouping devices into clusters is an essential means toward addressing these challenges. This can be evidenced by the fact that a set of devices forming a multicast group, when compared with the case of multiple D2D links working simultaneously, can reduce the sum interference associated with cellular users. Moreover, multicast can be more efficient than multiple unicasts in terms of energy, due to the broadcasting feature of wireless communications. As a representative example of $\mathrm{D} 2 \mathrm{D}$ clustering, a content distribution architecture, known as Collaborative Mobile Cloud (CMC), is proposed in [2]. A CMC consists of mobile terminals that are in need of the same content and are geographically close. Through a CMC, the same content requested by multiple mobile terminals can be spilt into several segments. For each of these segments, the base station (BS) only needs to send it to one of the mobile terminals once. These mobile terminals, after receiving their own content segments, can share the segments by sending them around within the CMC. 
On the other hand, simultaneous wireless information and power transfer (SWIPT) has emerged as a powerful mechanism against the energy depletion in wireless communications. SWIPT admits two architectures-time switching and power splitting. In the former, the receiver periodically switches between information decoding and energy harvesting $(\mathrm{EH})$ modules. In the latter, the receiver separates the received signals into two streams, one for the energy harvester and the other for the information decoder. Recent studies show the ndication that SWIPT can be expected to be fully used in D2D communications.

While the SWIPT realizes the energy harvesting from information signal streams, SWIPTenabled D2D communication scenarios have extensively explored. Huang et al. [3] surveyed the state-of-the-art of SWIPT architectures and enabling technologies, identified technical challenges to implement SWIPT, and showcased a novel SWIPT-supported D2D power allocation mechanism to illustrate the importance of the application of the SWIPT technology. Kaur et al. [4] proposed a network model for the performance analysis of D2D communications in a peer-to-peer SWIPT strategy with RF transmitters. Also, a joint power control and resource allocation problem in SWIPT-based energy-harvesting D2D underlay networks was investigated in [5]. Furthermore, Ali el at. [6] employed the SWIPT energy harvesting technique at the relay nodes to prolong the lifetime of energy-constrained networks and integrated D2D clustering techniques into cellular networks so that communication services can be effectively maintained when the cellular infrastructure becomes partially dysfunctional. By analyzing the above research work, the D2D cluster and SWIPT technology are not effectively combined to improve communication energy efficiency. It is worth noting that the resource allocation problem in CMC-assisted and SWIPT-enabled D2D content sharing scenarios was investigated in [7], and the EE of the entire system could be improved to a certain extent in that study. Unfortunately, the following critical issues were not addressed appropriately in [7].

1) How to segment the data contents?

2) In addition to spectrum utilization improvement, can a better EE be gained if the reuse mode of D2D communications is adopted?

3) How to guarantee the fairness of energy transfer and data transmission within the CMC?

4) What if SWIPT is supported by D2D user devices in terms of the system EE?

Dealing with these issues can contribute significantly toward enhancing the system EE, which is part of the motivation of our work.

In this paper, We propose an optimization framework for $\mathrm{CMC}$ in the content sharing scenario. The framework consists of three modules: water-filling-based data segmentation, resource allocation, and power control. In particular, SWIPT is considered in D2D communications within the $\mathrm{CMC}$, so that the lifetime of mobile devices is prolonged. The main contributions of our work are summarized below.

1) Relying on the optimal beamforming vector for each mobile terminal obtained by a traditional method, we devise a water-filling-based data segmentation approach that fully makes use of channel conditions for content distribution.

2) We consider SWIPT-empowered D2D links and formulate the resource allocation and the related power control problems in terms of the energy optimization. Following the proposal of an efficient algorithm that computes the optimal resources allocation for D2D links, a nonlinear programming method is utilized to solve the power control problem, and the ensuing power control algorithm is exhibited as an optimizer for the system energy efficiency.

3) To demonstrate the validity of our proposed framework, we compare our scheme against four variations of it through extensive simulations. The results indicate that the 
system EE of our scheme dominates that of the other four, confirming the effectiveness of our design.

The rest of this paper is organized as follows. Section 2 presents the communication framework in which our study is carried out. Section 3 formulates the problems that are intended to be investigated in this paper, and Section 4 subsequently proposes our energy-efficient scheme/solutions to these problems. Simulations are conducted in Section 5 to validate the proposed solutions, and Section 6 concludes the paper.

\section{System Model}

\subsection{System Overview}

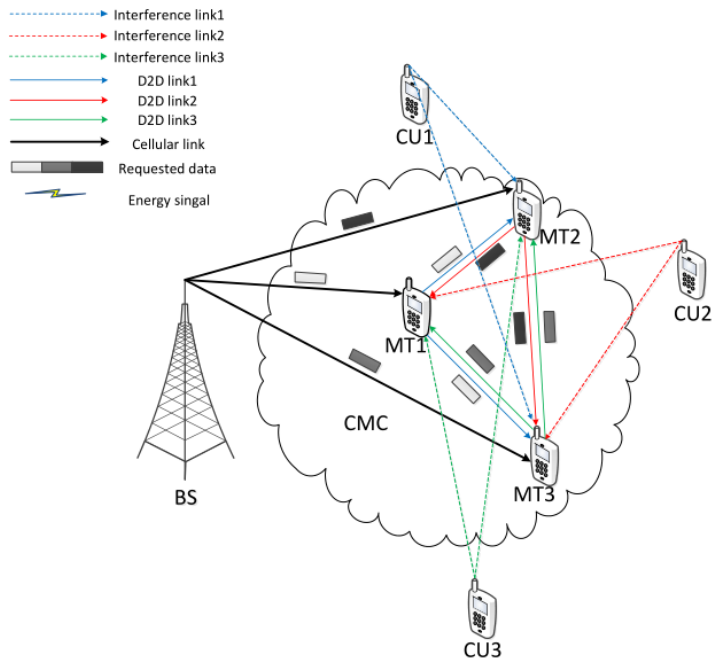

Fig. 1. An example of the communication scenario

A CMC-assisted and SWIPT-enabled communication scenario is considered. In this scenario, there are $K$ mobile terminals (MTs) reusing the uplink resources of $M(M \geq K)$ cellular users for content sharing, and a base station (BS) equipped with $Y$ antennas. All mobile terminals are assumed to be able to decode information and harvest energy from signals simultaneously. Also, the BS can accurately obtain the channel state information for all devices. Fig. 1 depicts an illustrative example of the considered scenario, in which three mobile terminals MT1, MT2, and MT3 request the same content from the BS. MT1, MT2, and MT3 reuse the uplink resource of cellular users CU1, CU2, and CU3, respectively. The BS precaches the content, divides the content into three blocks, and sends them to the corresponding MTs. The MTs in the CMC share the received data blocks with each other via D2D communications. Note that a D2D link in the CMC, on one hand, is interfered by the associated cellular user; on the other hand, receives the energy transmitted from the BS. As MTs in CMC can harvest energy 
by virtue of SWIPT, they will eventually receive the whole content and make full use of transmit power and interference for energy harvesting.

\subsection{Energy Consumption of Cellular Communication}

The data transmitted to MT $k$ from the BS can be expressed as

$$
\mathbf{x}_{k}=\boldsymbol{\omega}_{k} a_{k}
$$

where the complex vector $\boldsymbol{\omega}_{k} \in \mathrm{C}^{Y \times 1}$ is the beamforming vector associated with the BS and MT $k, a_{k}$ is the source information signal emitted from the BS. As such, the signal received by MT $k$ can be expressed as

$$
y_{k}=\mathbf{h}_{B, k} \mathbf{x}_{k}+n,
$$

where $\mathbf{h}_{B, k} \in \mathrm{C}^{1 \times Y}$ denotes the downlink channel coefficient from the BS to MT $k$, and $n$ is the additive Gaussian noise which follows the normal distribution $N\left(0, \sigma^{2}\right)$.

Therefore, the data rate between the BS and MT $k$ can be calculated as

$$
R_{B, k}=B W \cdot \log _{2}\left(1+\frac{\left|\mathbf{h}_{B, k} \omega_{k}\right|^{2}}{\sigma^{2}}\right)
$$

where $B W$ is the associated bandwidth of the cellular communication. Thus, the energy consumed at the BS for transmitting data to all MTs would be.

$$
E C_{B}^{\mathrm{Tx}}=\sum_{k=1}^{K} \operatorname{Tr}\left(\boldsymbol{\omega}_{k} \boldsymbol{\omega}_{k}^{\mathrm{H}}\right) \cdot \frac{S_{k}}{R_{B, k}},
$$

where $\operatorname{Tr}(\cdot)$ denotes the trace of a matrix, $(\cdot)^{\mathrm{H}}$ is the Hermitian transpose of a matrix, and $S_{k}$ is the size of the data that MT $k$ receives and $\sum_{k=1}^{K} S_{k}=S$ (the total size of data received by all MTs).

\subsection{Energy Consumption of D2D Communications within CMC}

Since D2D communications within the CMC take place by reusing the uplink resources of cellular users, we use $\rho_{k, m}$ to represent the status of resource allocations for D2D communications. Specifically,

$$
\rho_{k, m}=\left\{\begin{array}{ll}
1, & \text { if CU } m \text { 's resource is reused by MT } k \\
0, & \text { otherwise }
\end{array} .\right.
$$

So, the data rate of the D2D communication with MT $k$ being the transmitter would be 


$$
R_{k}=\sum_{m=1}^{M} B W \cdot \log _{2}\left(1+\gamma_{k, m}\right) \rho_{k, m},
$$

Where

$$
\gamma_{k, m}=\min _{k^{\prime} \in\{1,2, \cdots, K\}, k^{\prime} \neq k}\left\{\frac{(1-\theta) P_{\mathrm{k}}^{\mathrm{Tx}}\left|h_{k, k^{\prime}}\right|^{2} d_{k, k^{\prime}}^{-\alpha}}{(1-\theta)\left(P_{m}^{\mathrm{Tx}}\left|h_{m, k^{\prime}}\right|^{2} d_{m, k^{\prime}}^{-\alpha}+\sigma^{2}\right)+\sigma_{s p}^{2}}\right\},
$$

is the SINR of the transmitter MT $k ; h_{k, k^{\prime}}$ is the channel fading coefficient between two different mobile terminals MT $k$ and MT $k^{\prime} ; h_{m, k^{\prime}}$ is the channel fading coefficient between CU $m$ and MT $k^{\prime} ; \alpha$ is the path loss exponent of D2D communications; $P_{m}^{\mathrm{Tx}}$ and $P_{k}^{\mathrm{Tx}}$ are the transmit power of CU $m$ and MT $k$, respectively; $d_{k, k^{\prime}}$ and $d_{m, k^{\prime}}$ are the distance form MT $k$ to MT $k^{\prime}$ and from CU $m$ to MT $k^{\prime}$, respectively; $\theta$ is the power-splitting coefficient; and $\sigma_{s p}^{2}$ is the signal processing noise power.

Note that in D2D communications, the SINR of a D2D receiver is typically greater than the preset threshold. So, the signals received at any D2D receiving end can be split into two streams, with one stream being used for the default purpose (to meet the minimum SINR requirement) and the other for energy harvesting. As such, for the multiple D2D communications with MT $k$ being the transmitter, the energy consumption in these communications can be calculated as

$$
E C_{k}^{\mathrm{Tx}}=\left(P_{k}^{\mathrm{Tx}}+(K-1) P^{\mathrm{Rc}}\right) \cdot \frac{S_{k}}{R_{k}},
$$

where $P^{\mathrm{Rc}}$ is the power consumption of a receiver MT in the CMC, and the energy harvested by all the receivers in the $\mathrm{CMC}$ would be

$$
E H_{-k}=\zeta \theta \sum_{m=1}^{M} \sum_{k^{\prime}=1, k^{\prime} \neq k}^{K}\left\{\left[P_{x}^{\mathrm{Tx}}\left|h_{k, k^{\prime}}\right|^{2} d_{k, k^{\prime}}^{-\alpha}+P_{m}^{\mathrm{Tx}}\left|h_{m, k^{\prime}}\right|^{2} d_{m, k^{\prime}}^{-\alpha}+\sigma^{2}\right] \cdot \frac{S_{k}}{R_{k}} \cdot \rho_{k, m}\right\},
$$

where $\zeta$ is the energy conversion efficiency indicator.

\section{Problem Formulation}

After obtaining the optimal beamforming vector $\boldsymbol{\omega}_{k}^{*}$, for $\forall k$ using technique from [8], we can formulate the problem of minimizing cellular communication energy consumption as (P1),

$$
\min _{S_{k}} E C_{B}^{\mathrm{Tx}}=\sum_{k=1}^{K} \operatorname{Tr}\left(\boldsymbol{\omega}_{k}^{*} \boldsymbol{\omega}_{k}^{* \mathrm{H}}\right) \cdot \frac{S_{k}}{R_{B, k}},
$$


s.t.

$$
\begin{aligned}
& C 1: \sum_{k=1}^{K} S_{k}=S, \\
& C 2: 0 \leq S_{k} \leq S,
\end{aligned}
$$

condition $\mathrm{C} 1$ ensures (indirectly) that each mobile terminal will eventually receive the entire content sent from the BS.

Considering that the $E H_{-k}$ eventually comes from $E C_{k}^{\mathrm{Tx}}$, we can, without loss of generality, assume that $E H_{-k}=\pi_{k} E C_{k}^{\mathrm{Tx}}$, where $\pi_{k}$ is the energy harvesting efficiency and $0<\pi_{k}<1$. As such, the problem of minimizing the energy consumption for D2D communications can be described as (P2),

$$
\min _{\mathbf{P}_{D}, \boldsymbol{\rho}} \varepsilon^{\prime}\left(\mathbf{P}_{D}, \boldsymbol{\rho}\right)=\sum_{k=1}^{K}\left(1-\pi_{k}\right) E C_{k}^{\mathrm{Tx}},
$$

s.t.

$$
\begin{aligned}
& C 3: P_{k}^{\mathrm{Tx}} \leq P_{D_{\max }^{\mathrm{Tx}}}^{\mathrm{Tx}} \text {, for } \forall k, \\
& C 4: \gamma_{k, m} \geq \gamma_{D}, \text { for } \forall k, \\
& C 5: \sum_{m=1}^{M} \rho_{k, m}=1, \text { for } \forall k, \\
& C 6: \sum_{k=1}^{K} \rho_{k, m} \leq 1, \text { for } \forall k,
\end{aligned}
$$

where

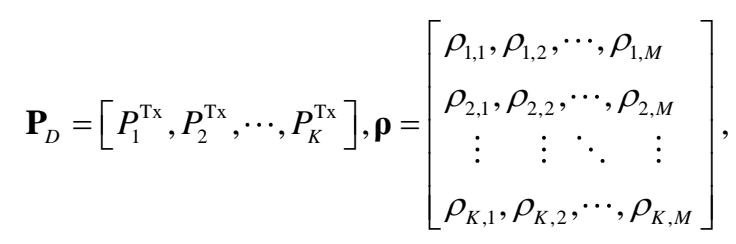

Condition C3 stipulates that the transmit power of any mobile terminal must not exceed its upper bound; condition $\mathrm{C} 4$ states that the minimum SINR of D2D communications must besatisfied for any D2D link; condition C5 guarantees that each 2D link will be allocated a channel; condition C6 requires that a single channel cannot be allocated to two or more D2D links at the same time.

\section{An Energy-Efficient Communication Scheme}

In this section, we present our proposed energy-efficient communication scheme, which includes three modules: water-filling-based data segmentation, resource allocation, and power control mechanisms.

\subsection{Water-filling-based Data Segmentation}


Note that in order to minimize energy consumption of cellular communications, a larger amount data segments should be transmitted over better quality cellular links. Hence, we use a water-filling-based approach to solve problem (P1).

In light of the constraint of problem (P1) $\sum_{k=1}^{K} S_{k}=S$, we should arrange a larger data segment to cellular link between the BS and MT $k$ if $\frac{R_{B, k}}{\operatorname{Tr}\left(\omega_{k}^{*} \boldsymbol{\omega}_{k}^{* \mathrm{H}}\right)}$ is higher. Hence,

$$
S_{k}=S \frac{R_{B, k} / \operatorname{Tr}\left(\boldsymbol{\omega}_{k}^{*} \boldsymbol{\omega}_{k}^{* \mathrm{H}}\right)}{\sum_{k=1}^{K}\left(R_{B, k} / \operatorname{Tr}\left(\boldsymbol{\omega}_{k}^{*} \boldsymbol{\omega}_{k}^{* \mathrm{H}}\right)\right)}
$$

\subsection{Resource Allocation for D2D Communications}

Now we tackle problem (P2). From what discussed in preceding sections, we know

$$
\begin{aligned}
E C_{k}^{\mathrm{Tx}} & =\left(P_{k}^{\mathrm{Tx}}+(K-1) \cdot P^{\mathrm{Rc}}\right) \cdot \frac{S_{k}}{R_{k}} \\
& =\left(P_{k}^{\mathrm{Tx}}+(K-1) \cdot P^{\mathrm{Rc}}\right) \cdot \frac{S_{k}}{\sum_{m=1}^{M} B W \cdot \log _{2}\left(1+\gamma_{k, m}\right) \rho_{k, m}}
\end{aligned}
$$

Before optimal $\left(P_{k}^{\mathrm{Tx}}\right)^{*}$ is obtained, we can assume, without loss of generality, that $P_{k}^{\mathrm{Tx}}$ is a constant for all MT $k$, as it will not affect the final optimization result. In order to minimize $\sum_{k=1}^{K} E C_{k}^{\mathrm{Tx}}$, we can maximize the expression below,

$$
\sum_{k=1}^{K} \sum_{m=1}^{M} B W \cdot \log _{2}\left(1+\gamma_{k, m}\right) \rho_{k, m}
$$

As such, we design an efficient resource allocation algorithm to maximize (17). The main idea of the algorithm lies in how to maximize the sum of SINRs for D2D communications within the $\mathrm{CMC}$, and it is described as follows:

Step1: Renumber all the mobile terminals by the descending order of their distance to the BS such that $d_{B, 1} \geq d_{B, 2} \geq \cdots \geq d_{B, K-1} \geq d_{B, K}$. Let $\rho_{k, 0}$ indicates whether the MT $k$ has been assigned the resource, and $\operatorname{Ind}_{m} \in\{1,2, \cdots, K\}$ indicates to which mobile terminal the resource of CU $k$ is being reused. Make the following initializations: $\rho_{k, 0}=0$, for $\forall k \in\{1,2, \cdots, K\}$; Ind $_{m}=0$, for $\forall m \in\{1,2, \cdots, M\} ; \rho_{k, m}=0$, for $\forall k \in\{1,2, \cdots, K\}, \forall m \in\{1,2, \cdots, M\}$; and calculate $\gamma_{k, m}$ for all $k$ and $m$.

Step2: To each $k \in\{1,2, \cdots, K\}$, find $m^{*}=\arg \max \left\{\gamma_{k, m}\right\}$, for $\forall m \in\{1,2, \cdots, M\}$. If Ind $_{m^{*}}=0$, let $\operatorname{Ind}_{m^{*}}=k$ and $\rho_{k, m^{*}}=1$. If $\operatorname{Ind}_{m^{*}} \neq 0$, say $\operatorname{Ind}_{m^{*}}=k^{*}$, then let $\gamma_{k, m^{\prime}}^{*}=\max _{m^{\prime}}\left\{\gamma_{k, m^{\prime}}\right\} \quad, \quad \gamma_{k^{*}, m^{\prime}}^{*}=\max _{m^{\prime}}\left\{\gamma_{k^{*}, m^{\prime}}\right\} \quad, \quad m^{\prime} \in\left\{1,2, \cdots, m^{*}-1, m^{*}+1, \cdots, M\right\} \quad$. If 
$\gamma_{k, m^{*}}-\gamma_{k, m^{\prime}}^{*}>\gamma_{k^{*}, m^{*}}-\gamma_{k^{*}, m^{\prime}}^{*}$, then let $\operatorname{Ind}_{m^{*}}=k, \rho_{k^{*}, m^{*}}=0$ and $\rho_{k, m^{*}}=1$. If $k<K, k$ will incremented by 1 , and the current step will be repeated; otherwise, go to step3.

Step3: For each $i \in\{1,2, \cdots, K\}$ and each $j \in\{1,2, \cdots, M\}$, if $\rho_{i, j}=1$, then let $\rho_{i, 0}=1$; for all $m \in\{1,2, \cdots, M\}, \gamma_{i, m}=0$; and for all $k \in\{1,2, \cdots, K\}, \gamma_{k, m}=0$.

Step4: Calculate the sum of $\rho_{k, 0}, k \in\{1,2, \cdots, K\}$. If $\sum_{k=1}^{K} \rho_{k, 0}=K$, then terminate the algorithm and obtain optimal $\boldsymbol{\rho}$. If $\sum_{k=1}^{K} \rho_{k, 0}<K$, let $k=1$ and go back to Step2.

Note that above algorithm, after no more than $K$ iterations of the whileloop, at least one D2D link will be allocated some resource. Also, any allocated resources and any D2D links that have acquired resource will not be involved in subsequent computations. Thus, all D2D links will eventually be allocated resource after at most $K^{2}$ iterations of the while-loop. Considering that the cost of finding the maximum of $\gamma_{k, m}$ is $M$ ineachiteration, the worst-case time complexity of above algorithm is evidently $O\left(K^{2} M\right)$. Note also that the above algorithm allows mobile terminals with a small index be allocated a better resource than those mobile terminals with a large index. The reason for our algorithm to work in this fashion is because mobile terminals with a small index are farther away from the BS than those with a large index, and the distance between a mobile terminal and the BS is the major measurement in determining the energy consumptions for BS to charge the mobile terminal (i.e., longer distance implies greater energy consumption). Thus, the outcome of our algorithm is a balanced and fair consideration of resource allocation.

\subsection{Power Control for D2D Communications}

Once all D2D links have been allocated resource, the transmit power of D2D transmitters need to be controlled or adjusted towards mitigating the energy consumption of D2D communications. Note that $(\mathrm{P} 2)$ can be equivalently regarded as $K$ independent subproblems (P3) as follows,

$$
\min _{P_{k}^{\mathrm{Tx}}} \varepsilon^{\prime}\left(P_{k}^{\mathrm{Tx}}\right)=\sum_{k=1}^{K}\left(1-\pi_{k}\right) E C_{k}^{\mathrm{Tx}},
$$

s.t.

$$
\begin{aligned}
& C 3: P_{k}^{\mathrm{Tx}} \leq P_{D_{\max }^{\mathrm{Tx}}}^{\mathrm{x}}, \\
& C 4: \frac{(1-\theta) P_{k}^{\mathrm{Tx}} h_{k, k^{*}}}{I_{k^{*}}} \geq \gamma_{D},
\end{aligned}
$$

where $m^{*}$ represents the index of the optimal resource allocation for MT $k$ being the D2D transmitter. $k^{*}=\underset{k^{\prime} \neq k}{\arg \min }\left\{\frac{(1-\theta) P_{k}^{\mathrm{Tx}}\left|h_{k, k^{\prime}}\right|^{2} d_{k, k^{\prime}}^{-\alpha}}{(1-\theta)\left(P_{m}^{\mathrm{Tx}}\left|h_{m, k^{\prime}}\right|^{2} d_{m, k^{\prime}}^{-\alpha}+\sigma^{2}\right)+\sigma_{s p}^{2}}\right\}, I_{k^{*}}$ represents the sum of the noise and the interference experienced at the receiver $k^{*}$. Toward solving the problem (P3), we transform it into a different form by using a nonlinear programming technique [9]. Specifically, let 


$$
U\left(P_{k}^{\mathrm{Tx}}\right)=S_{k}\left(P_{k}^{\mathrm{Tx}}+(K-1) P^{\mathrm{Rc}}\right),
$$

and,

$$
R\left(P_{k}^{\mathrm{Tx}}\right)=B W \cdot \log _{2}\left(1+\frac{(1-\theta) P_{k}^{\mathrm{Tx}} h_{k, k^{*}}}{I_{k^{*}}}\right)
$$

then we have,

$$
\varepsilon^{\prime}\left(P_{k}^{\mathrm{Tx}}\right)=\left(1-\pi_{k}\right) \frac{U\left(P_{k}^{\mathrm{Tx}}\right)}{R\left(P_{k}^{\mathrm{Tx}}\right)},
$$

Lemma1. Let $Q_{k}=\frac{U\left(P_{k}^{\mathrm{Tx}}\right)}{R\left(P_{k}^{\mathrm{Tx}}\right)}$. Then, $Q_{k}$ reaches its optimal value if and only if

$$
\min _{P_{k}^{\mathrm{Tx}}}\left\{U\left(P_{k}^{\mathrm{Tx}}\right)-Q_{k}^{*} \cdot R\left(P_{k}^{\mathrm{Tx}}\right)\right\}=0
$$

where $Q_{k}^{*}$ is the optimal value of $Q_{k}$.

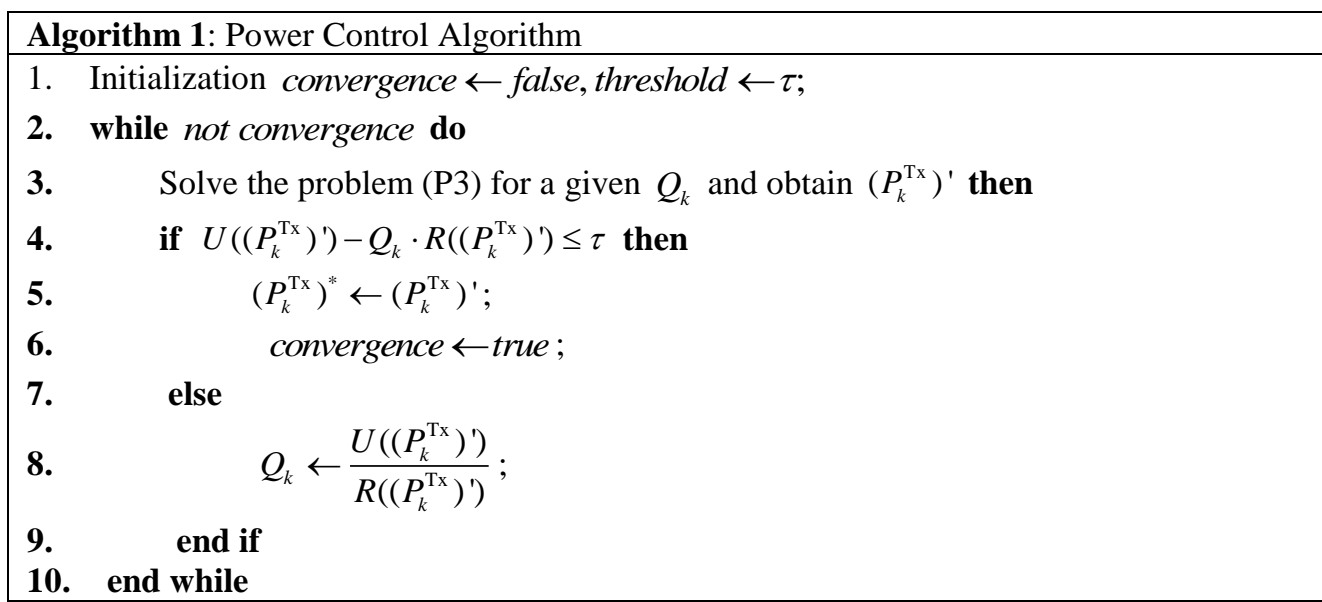

In order to find the optimal value of $P_{k}^{\mathrm{Tx}}$, we need to address the following problem (P4),

$$
\min _{P_{k}^{\mathrm{Tx}}}\left\{U\left(P_{k}^{\mathrm{Tx}}\right)-Q_{k}^{*} \cdot R\left(P_{k}^{\mathrm{Tx}}\right)\right\}
$$

s.t.

$$
\begin{aligned}
& C 3: P_{k}^{\mathrm{Tx}} \leq P_{D_{\max }^{\mathrm{Tx}}}^{\mathrm{Tx}}, \\
& C 4: \frac{(1-\theta) P_{k}^{\mathrm{Tx}} h_{k, k^{*}}}{I_{k^{*}}} \geq \gamma_{D} .
\end{aligned}
$$

It is proven that Problem (P4), as a convex problem, can be effectively solved by using the Karush-Kuhn-Tucker (KKT) conditions, which are the sufficient and necessary conditions for a convex problem to have an optimal solution. So we can obtain the optimal solution of problem 
(P4) $\left(P_{k}^{\mathrm{Tx}}\right)^{\prime}$. The entire process above can be characterized as an iterative algorithm, shown in Algorithm 1.

\section{Numerical Simulations}

In this section, extensive simulations are conducted todemonstrate the efficiency of the proposed scheme. Some parameters used in the simulations are set in Table 1 according to $3 \mathrm{GPP}^{[10]}$. For the purpose of performance evaluation, we define the energy consumption of the entire system to be

$$
E C=E C_{B}^{\mathrm{Tx}}+\sum_{k=1}^{K} E C_{k}^{\mathrm{Tx}}-\sum_{k=1}^{K} E H_{-k}
$$

and the delay of communcation system is defined as

$$
\text { Delay }=\max \left(\frac{S_{k}}{R_{B, k}}\right)+\max \left(\frac{S_{k}}{R_{k}}\right)
$$

Table 1. Parameter Settings.

\begin{tabular}{ll}
\hline Parameter & Value \\
\hline$P_{D_{\max }^{\mathrm{Tx}}}$ & $23 \mathrm{dbm}$ \\
$P_{B_{\max } \mathrm{Tx}}$ & $46 \mathrm{dbm}$ \\
$\zeta$ & 0.5 \\
$\theta$ & 0.5 \\
$\alpha$ & 2 \\
$S$ & $1 \mathrm{mbit}$ \\
$B W$ & $10 \mathrm{MHZ}$ \\
\hline
\end{tabular}

To evaluate the performance of our proposed communication scheme, we vary it into four different versions, and then compare the system delay and energy consumption against them.

Scheme 0: Our original mechanism.

Scheme 1: The data segmentation is modified in such a way that data is evenly divided into blocks. Other modules remain unchanged.

Scheme 2: The resource allocation algorithm is altered to a random resource allocation. Other modules remain unchanged.

Scheme 3: The power control mechanism is changed to a new one that maximizes the data rate of $\mathrm{D} 2 \mathrm{D}$ communications. Other modules remain unchanged.

Scheme 4: The SWIPT component is removed. That is, the MTs are not able to scavenge energy from signals during D2D communications. Other modules remain unchanged. 


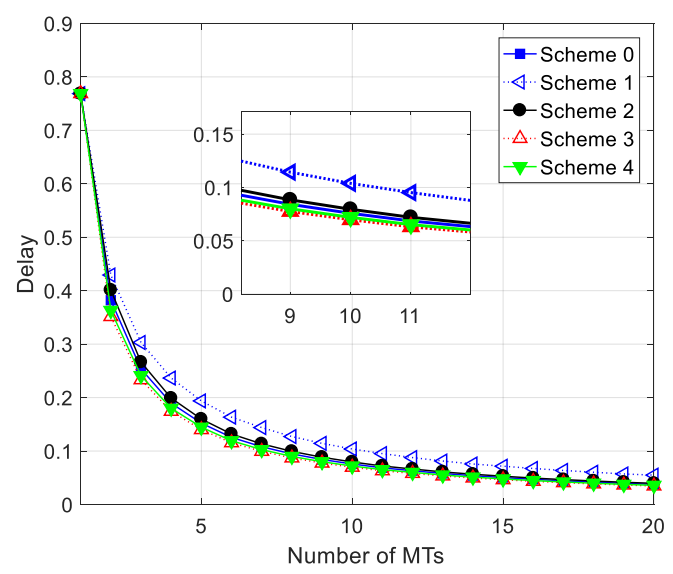

Fig. 2. Delay comparison.

The comparisons of our original mechanism with these four varied schemes in terms of delay and energy consumption are shown in Figs. 2 and 3. By observing Fig. 2, we notice that our proposed communication scheme, i.e., Scheme 0 , sits in the middle of the five schemes. In other words, the delay performance of Scheme 0 is better than that of Schemes 1 and 2, but worse than that of Schemes 3 and 4.

The reasons for this can be understood as follows. According to the definition of Schemes $1,2,3$, and 4, a water-filling based data segmentation method is used in Scheme 0, which is better than even-data-dividing used in Scheme 1 in the sense that channel condition is utilized in a more efficient way. As such, the delay of Scheme 0 is much lower than that of Scheme 1. Also, note that the resource allocation process in Scheme 0 maximizes the SINR of D2D communications in CMC, leading to an improved data rate of D2D commutations. Since this optimizing resource allocation component is changed to a random resource allocation in Scheme 2, some loss in data rate of D2D communications will be inevitable in Scheme 2, which will consequently result in a longer delay in Scheme 2. On the other hand, due to the utilization of a data-rate-maximizing power control technique in Scheme 3, Scheme 3 has the best D2D communication data rate among the five schemes. As a result, Scheme 3 has the lowest delay. Regarding Scheme 4, note that it does not include the SWIPT technology as part of its components, as opposed to Scheme 0 which does. Since the support for the SWIPT technology may decrease the SINR/data rate of D2D communications in the CMC slightly, we see that the delay of Scheme 0 is worse than that of Scheme 4. 


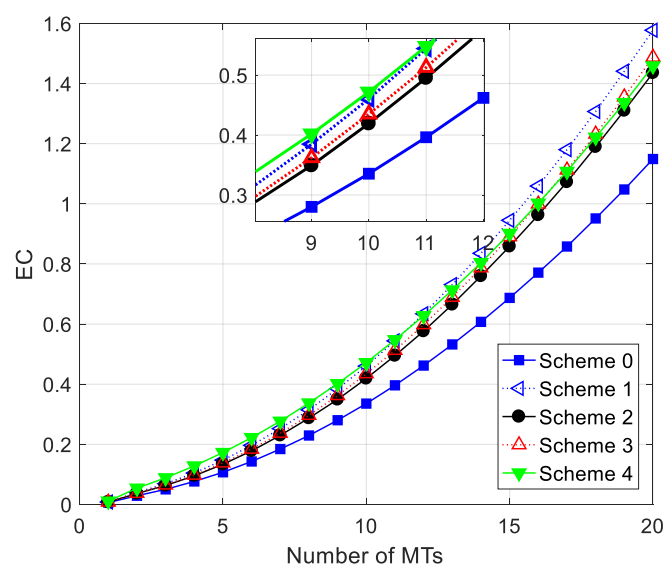

Fig. 3. EC comparison.

Although the delay of Scheme 0 is not the best among the five schemes, its EC is the best as shown in Fig. 3. The result in this figure further confirms the effectiveness of the design of our optimization framework.

\section{Conclusion}

In this work, we have investigated the energy efficiency problem for content sharing with Collaborative Mobile Clouds. We have proposed an energy-efficient communication scheme, which is made up of three modules: water-filling-based data segmentation, D2D communication resource allocation, and power control. In particular, for D2D communications within the CMC, SWIPT-empowered D2D transmissions have been considered, both resource allocation and power control have been designed. We have also compared our scheme against four variations of it, and the results of comparisons indicate that the system EE of our proposed scheme dominates that of the other four, confirming the effectiveness of our design. 


\section{References}

[1] Y. Li, J. Liu, B. Cao, and C. Wang, "Joint optimization of radio and virtual machine resources with uncertain user demands in mobile cloud computing," IEEE Transactions on Multimedia, vol. 20, no. 9, pp. 2427-2438, Sept 2018.

[2] M. V. Pedersen and F. H. P. Fitzek, "Mobile clouds: The new content distribution platform," Proceedings of the IEEE, vol. 100, no. Special Centennial Issue, pp. 1400-1403, May 2012.

[3] J. Huang, C. Xing, and C. Wang, "Simultaneous wireless information and power transfer: Technologies, applications, and research challenges," IEEE Communications Magazine, vol. 55, no. 11, pp. 26-32, Nov 2017.

[4] V. Kaur and S. Thangjam, "A stochastic geometry analysis of rf energy harvesting based $\mathrm{d} 2 \mathrm{~d}$ communication in downlink cellular networks," in 2016 1st India International Conference on Information Processing (IICIP), Aug 2016, pp. 1-5.

[5] Z. Zhou, C. Gao, C. Xu, T. Chen, D. Zhang, and S. Mumtaz, "Energy-efficient stable matching for resource allocation in energy harvesting-based device-to-device communications," IEEE Access, vol. 5, pp. 15184-15196, 2017.

[6] K. Ali, H. X. Nguyen, Q. Vien, P. Shah, and Z. Chu, "Disaster management using d2d communication with power transfer and clustering techniques," IEEE Access, vol. 6, pp. 14643-14654, 2018.

[7] Z. Chang, J. Gong, Y. Li, Z. Zhou, T. Ristaniemi, G. Shi, Z. Han, and Z. Niu, "Energy efficient resource allocation for wireless power transfer enabled collaborative mobile clouds," IEEE Journal on Selected Areas in Communications, vol. 34, no. 12, pp. 3438-3450, Dec 2016.

[8] M. Zhu and S. Martinez, "An approximate dual subgradient algorithm for multi-agent non-convex optimization," IEEE Transactions on Automatic Control, vol. 58, no. 6, pp. 1534-1539, June 2013.

[9] W. Dinkelbach, "On nonlinear fractional programming," Management Science, vol. 13, pp. 492498, 1967.

[10] 3GPP. TR 36.746. Study on further enhancements to LTE Device to Device (D2D), UE to network relays for Internet of Things (IoT) and wearables [S]. 3rd Generation Partnership Project. 2018 